\title{
An Architecture for Generating Semantics-Aware Signatures
}

\author{
Vinod Yegneswaran Jonathon T. Giffin Paul Barford Somesh Jha \\ Computer Sciences Department \\ University of Wisconsin, Madison \\ \{vinod,giffin,jha,pb\}@cs.wisc.edu
}

\begin{abstract}
Identifying new intrusions and developing effective signatures that detect them is essential for protecting computer networks. We present Nemean, a system for automatic generation of intrusion signatures from honeynet packet traces. Our architecture is distinguished by its emphasis on a modular design framework that encourages independent development and modification of system components and protocol semantics awareness which allows for construction of signatures that greatly reduce false alarms. The building blocks of our architecture include transport and service normalization, intrusion profile clustering and automata learning that generates connection and session aware signatures. We demonstrate the potential of Nemean's semantics-aware, resilient signatures through a prototype implementation. We use two datasets to evaluate the system: (i) a production dataset for false-alarm evaluation and (ii) a honeynet dataset for measuring detection rates. Signatures generated by Nemean for NetBIOS exploits had a $0 \%$ false-positive rate and a $0.04 \%$ false-negative rate.
\end{abstract}

\section{Introduction}

Computer network security is a multidimensional activity that continues to grow in importance. The prevalence of attacks in the Internet and the ability of selfpropagating worms to infect millions of Internet hosts has been well documented [30,34]. Developing techniques and tools that enable more precise and more rapid detection of such attacks presents significant challenges for both the research and operational communities.

Network-security architectures often include network intrusion detection systems (NIDS) that monitor packet traffic between networks and raise alarms when malicious activity is observed. NIDS that employ misusedetection compare traffic against a hand-built database of signatures or patterns that identify previously docu- mented attack profiles $[3,18]$. While the effectiveness of a misuse-detector is tightly linked to the quality of its signature database, competing requirements make generating and maintaining NIDS signatures difficult. On one hand, signatures should be specific: they should only identify the characteristics of specific attack profiles. The lack of specificity leads to false alarms - one of the major problems for NIDS today. For example, Sommer and Paxson argue that including context, such as the victim's response, in NIDS signatures reduces false alarm rates [28]. On the other hand, signatures should be general so that they match variants of specific attack profiles. For example, a signature that does not account for transport or application-level semantics can lead to false alarms $[6,22,32]$. Thus, a balance between specificity and generality is an important objective for signatures.

We present the design and implementation of an architecture called Nemean ${ }^{1}$ for automatic generation of signatures for misuse-detection. Nemean aims to create signatures that result in lower false-alarm rates by balancing specificity and generality. We achieve this balance by including semantics awareness, or the ability to understand session-layer and application-layer protocol semantics. Examples of session layer protocols include NetBIOS and RPC, and application layer protocols include SMB, TELNET, NTP and HTTP. Increasingly, preprocessors for these protocols have become integral parts of NIDS. We argue that these capabilities are essential for automatic signature generation systems for the following reasons:

1. Semantics awareness enables signatures to be generated for attacks in which the exploit is a small part of the entire payload.

2. Semantics awareness enables signatures to be generated for multi-step attacks in which the exploit does not occur until the last step.

3. Semantics awareness allows weights to be assigned 
to different portions of the payload (e.g., timestamps, sequence numbers, or proxy-cache headers) based upon their significance.

4. Semantics awareness helps produce generalized signatures from a small number of input samples.

5. Semantics awareness results in signatures that are easy to understand and validate.

Our architecture contains two components: a data $a b$ straction component that normalizes packets from individual sessions and renders semantic context and a signature generation component that groups similar sessions and uses machine-learning techniques to generate signatures for each cluster. The signatures produced are suitable for deployment in a NIDS [3, 18, 31]. We address specificity by producing both connection-level and session-level signatures. We address generality by learning signatures from transport-normalized data and consideration of application-level semantics that enables variants of attacks to be detected. Therefore, we argue that Nemean generates balanced signatures. At present, Nemean's goal is to provide an automated mechanism to build accurate signatures that keep pace with exploits and network viruses released everyday and is not meant to "automate real-time deployment" of signatures. We discuss this issue in greater detail in Section 3.3.

The input to Nemean is a set of packet traces collected from a honeynet deployed on an unused IP address space. Any data observed at a honeynet [7] ${ }^{2}$ is anomalous, thus mitigating both the problem of privacy and the problem of separating malicious and normal traffic. ${ }^{3}$ We assume that the honeynet is subject to the same attack traffic as standard hosts and discuss the ramifications of this assumption in Section 8.

To evaluate Nemean's architecture, we developed a prototype implementation of each component. This implementation enables automated generation of signatures from honeynet packet traces. We also developed a simple alert generation tool for off-line analysis, which compares packet traces against signatures. While we demonstrate that our current implementation is extremely effective, the modular design of the architecture enables any of the individual components to be easily replaced. We expect that further developments will tune and expand individual components resulting in more timely, precise and effective signatures. From a broader perspective, we believe that our results demonstrate the importance of Nemean's capability in a comprehensive security architecture. Section 3 describes the architecture and Sections 4 and 5 present our prototype implementation of Nemean.

We performed two evaluations of our prototype. First, we calculated detection and misdiagnosis counts using packet traces collected at two unused /19 address ranges (16K total IP addresses) from two distinct Class B networks allocated to our campus. We collected sessionlevel data for exploits targeting ports 80 (HTTP), 139 and 445 (NetBIOS/SMB). Section 6 describes the data collection environment. We use this packet trace data as input to Nemean to produce a comprehensive signature set for the three target ports. In Section 7, we describe the major clusters and the signatures produced from this data set. Leave-out testing results indicate that our system generates accurate signatures for most common intrusions, including Code Red, Nimda, and other popular exploits. We detected $100 \%$ of the HTTP exploits and $99.96 \%$ of the NetBIOS exploits with 0 misdiagnoses. Next, we validated our signatures by testing for false alarms using packet traces of all HTTP traffic collected from our department's border router. Nemean produced 0 false alarms for this data set. By comparison, Snort [3] generated over 1,000 false alarms on the same data set. These results suggest that even with a much smaller signature set, Nemean achieves detectability rates on par with Snort while identifying attacks with superior precision and far fewer false alarms.

\section{Related Work}

Sommer and Paxson [28] proposed adding connectionlevel context to signatures to reduce false positives in misuse-detection. Handley et al. described transportlevel evasion techniques designed to elude a NIDS as well as normalization methods that disambiguate data before comparison against a signature [6]. Similar work described common HTTP evasion techniques and standard URL morphing attacks [22]. Vigna et al. [32] described several mutations and demonstrated that two widely deployed misuse-detectors were susceptible to such mutations. The works of Handley et al. and Vigna et al. highlight the importance of incorporating semantics into the signature generation process.

Honeypots are an excellent source of data for intrusion and attack analysis. Levin et al. described how honeypots extract details of worm exploits that can be analyzed to generate detection signatures [13]. Their signatures were generated manually.

Several automated signature generation systems have been proposed. Table 1 summarizes the differences between Nemean and the other signature-generation systems. One of the first systems proposed was Honeycomb developed by Kreibich and Crowcroft [11]. Like Nemean, Honeycomb generated signatures from traffic observed at a honeypot via its implementation as a Honeyd [20] ${ }^{4}$ plugin. At the heart of Honeycomb is the longest common substring (LCS) algorithm that looks for the longest shared byte sequences across pairs of con- 


\begin{tabular}{|l|c|c|c|c|c|}
\hline & Traffic source & $\begin{array}{c}\text { Generates Contextual } \\
\text { Signatures }\end{array}$ & $\begin{array}{c}\text { Semantics } \\
\text { Aware }\end{array}$ & $\begin{array}{c}\text { Signature Generation } \\
\text { Algorithm }\end{array}$ & $\begin{array}{c}\text { Target } \\
\text { Attack Class }\end{array}$ \\
\hline Nemean & Honeypots & $\begin{array}{c}\text { Yes (Generates connection- and } \\
\text { session- level signatures) }\end{array}$ & Yes & $\begin{array}{c}\text { (MSG) Clustering } \\
\text { and automata learning }\end{array}$ & General \\
\hline Autograph & DMZ & $\begin{array}{c}\text { No (Generates } \\
\text { byte-level signatures) }\end{array}$ & No & $\begin{array}{c}\text { (COPP) partitioning } \\
\text { content blocks }\end{array}$ & Worm \\
\hline Earlybird & DMZ & $\begin{array}{c}\text { No (Generates } \\
\text { byte-level signatures) }\end{array}$ & No & $\begin{array}{c}\text { Measuring } \\
\text { packet-content prevalence }\end{array}$ & Worm \\
\hline Honeycomb & Honeypots & $\begin{array}{c}\text { No (Generates } \\
\text { byte-level signatures) }\end{array}$ & No & General \\
\hline
\end{tabular}

Figure 1: Comparison of Nemean to other signature-generation systems.

nections. However, since Honeycomb does not consider protocol semantics, its pairwise LCS algorithm outputs a large number of signatures. It is also frequently distracted by long irrelevant byte sequences in packet payloads, thus reducing its capability for identifying attacks with small exploit strings, exemplified in protocols such as NetBIOS. We discuss this in greater detail in Section 7.4.

Kim and Karp [10] described the Autograph system for automated generation of signatures to detect worms. Unlike Honeycomb and Nemean, Autograph's input are packet traces from a DMZ that includes benign traffic. Content blocks that match "enough" suspicious flows are used as input to COPP, an algorithm based on Rabin fingerprints that searches for repeated byte sequences by partitioning the payload into content blocks. Like Honeycomb, Autograph does not consider protocol semantics. We argue that such approaches, while attractive in principle, seem viable for a rather limited spectrum of observed attacks and are prone to false positives. This also makes Autograph more susceptible to mutation attacks $[6,22,32]$. Finally, unlike byte-level signatures produced by Autograph, Nemean can produce both connection-level and session-level signatures.

Another system developed to generate signatures for worms, Earlybird [27], measured packet-content prevalence at a single monitoring point such as a network DMZ. By counting the number of distinct sources and destinations associated with strings that repeat often in the payload, Earlybird distinguished benign repetitions from epidemic content. Like Autograph, Earlybird also produced byte-level signatures and was not aware of protocol semantics. Hence Earlybird has the same disadvantages compared to Nemean as Autograph.

Pouget and Dacier [19] analyzed honeypot traffic to identify root causes of frequent processes observed in a honeypot environment. They first organized the observed traffic based on the port sequence. Then, the data was clustered using association-rules mining [1]. The resulting clusters were further refined using "phrase distance". Pouget and Dacier's technique is not semantics aware.

Julisch [8] also clustered alarms for the purpose of discovering the root-cause of an alarm. After clustering the alarms, Julisch's technique generated a generalized alarm for each cluster. Intuitively, generation of generalized alarms is similar to the automata-learning step of our algorithm. However, the goals and techniques used in our work are different than the ones used by Julisch.

In [4], Christodorescu et al. presented a semanticsaware methodology to detect malicious traits in $\mathrm{x} 86 \mathrm{bi}$ naries. Their approach is semantics aware because their algorithm incorporates semantics of x 86 instructions that are executed. In contrast, Nemean incorporates semantics of various protocols in parsing application level packet content. Hence, the malware-detection algorithm presented in [4] and the signature-generation algorithm of Nemean consider semantics at different levels.

Anomaly detection is an alternative approach for malicious traffic identification in a NIDS. Anomaly detectors construct a model of acceptable behavior and then flag any deviations from the model as suspicious. Anomalydetection techniques for detecting port scans have been explored in $[9,29]$. Balancing specificity and generality has proven extraordinarily difficult in anomaly-detection systems, and such systems often have a high false-alarm rate. This paper focuses on misuse-detection, and we will not discuss anomaly-detecting techniques further.

\section{Nemean Architecture}

As shown in Figure 2, Nemean's architecture is divided into two components: the data abstraction component and the signature generation component. The input to Nemean is a packet trace collected from a honeynet. Even when deployed on a small address space (e.g., a /24 containing 256 IP addresses), a honeynet can provide a large volume of data without significant privacy or false positives concerns.

\subsection{Data Abstraction Component}

The Data Abstraction Component (DAC) aggregates and transforms the packet trace into a well-defined data structure suitable for clustering by a generic clustering module without specific knowledge of the transport protocol or application-level semantics. We call these aggregation units semi-structured session trees (SSTs). The 


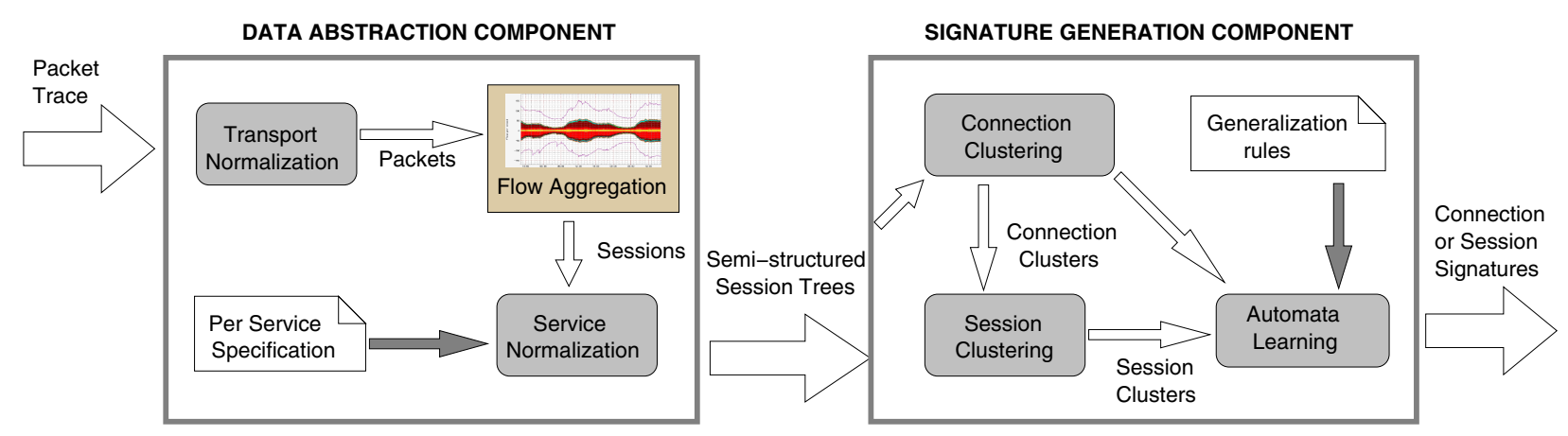

Figure 2: Components and data flow description of the Nemean architecture.

components of the DAC can then be thought of in terms of the data flow through the module as shown in Figure 2. While we built our own DAC module, in principle it could be implemented as an extension to a standard NIDS, such as a Bro policy script [18].

Transport normalization disambiguates obfuscations at the network and transport layers of the protocol stack. Our DAC reads packet traces through the libpcap library. This can either be run online or offline on tcpdump traces. This step considers transport-specific obfuscations like fragmentation reassembly, duplicate suppression, and checksums. We describe these in greater detail in Section 4.

The aggregation step groups packet data between two hosts into sessions. The normalized packet data is first composed and stored as flows. Periodically, the DAC expires flows and converts them into connections. A flow might be expired for two reasons: a new connection is initiated between the same pair of hosts and ports or the flow has been inactive for a time period greater than a user defined timeout ( 1 hour in our experimental setup). Flows are composed of packets, but connections are composed of request-response elements. Each connection is stored as part of a session. A session is a sequence of connections between the same host pairs.

Service-specific information in sessions must be normalized before clustering for two reasons. First, classification of sessions becomes more robust and clustering algorithms can be independent of the type of service. Second, the space of ambiguities is too large to produce a signature for every possible encoding of attacks. By decoding service-specific information into a canonical form, normalization enables generation of a more compact signature set. A detection system must then first decode attack payloads before signature matching. This strategy is consistent with that employed by popular NIDS [3]. We describe the particular normalizations performed in greater detail in Section 4.

The DAC finally transforms the normalized sessions into XML-encoded SSTs suitable for input to the clustering module. This step also assigns weights to the elements of the SST to highlight the most important attributes, like the URL in an HTTP request, and deemphasize the less important attributes, such as encrypted fields and proxy-cache headers in HTTP packets. Nemean's current weight assignment is simply based on our expert knowledge of protocols and prevalent attacks. It should be noted that the weights are not tuned to reflect a specific attack, but are meant to be sufficiently general and reflect high level behavior drawn from a large class of attacks. We expect that these might need to periodically adjusted to accommodate significant changes in exploit patterns.

\subsection{Signature-Generation Component}

The clustering module groups sessions and connections with similar attack profiles according to a similarity metric. We assume that sessions grouped together will correspond to a single attack type or variants of a well-known attack while disparate clusters represent distinct attacks or attack variants that differ significantly from some original attack. Effective clustering requires two properties of the attack data. First, data that correspond to an attack and its variants should be measurably similar. A clustering algorithm can then classify such data as likely belonging to the same attack. Second, data corresponding to different attacks must be measurably dissimilar so that a clustering algorithm can separate such data. We believe that the two required properties are unlikely to hold for data sets that include significant quantities of nonmalicious or normal traffic. Properties of normal traffic vary so greatly as to make effective clustering difficult without additional discrimination metrics. Conversely, malicious data contains identifiable structure even in the presence of obfuscation and limited polymorphism. Nemean's use of honeynet data enables a reasonable number of meaningful clusters to be produced. While each cluster ideally contains the set of sessions or connections for some attack, we also presume that this data will contain minor obfuscations, particularly in the sequential structure of the data, that correspond to an attacker's at- 
tempts to evade detection. These variations provide the basis for our signature generation component.

The automata learning module constructs an attack signature from a cluster of sessions. A generator is implemented for a target intrusion detection system and produces signatures suitable for use in that system. This component has the ability to generate highly expressive signatures for advanced systems, such as regular expression signatures with session-level context that are suitable for Bro $[18,28]$. Clusters that contain many nonuniform sessions are of particular interest. These differences may indicate either the use of obfuscation transformations to modify an attack or a change made to an existing attack to produce a new variant. Our signature generation component generalizes these transformations to produce a signature that is resilient to evasion attempts. Generalizations enable signatures to match malicious sequences that were not observed in the training set.

\subsection{Current Limitations}

New worms, viruses, and variants of existing malware appear in the Internet everyday [16], and standard collections of signatures are not able to keep pace. Thus, the immediate goal for Nemean is to address this gap by automating signature generation. Nemean does not address automating the real-time deployment of signatures. Given our emphasis on accurate, efficient signatures and not on timeliness, the current Nemean design includes the following simple manual selection process:

- Selecting either or both of the generated session and connection-level signatures for a given cluster. For multistep attacks such a Welchia, there is a benign connection (a GET / request) that precedes the attack sequence. In this case, the operator simply chooses either the connection signatures for the following steps of Welchia and/or the session signature, but whitelists the signature corresponding to the benign first step. We provide results from both connection and session-level signatures for each attack in our evaluation but remove the benign connection corresponding to Welchia. This was not an issue for other attacks.

- A sanity check to ensure that a signature corresponds to an attack cluster and not a misconfiguration or intentional data pollution. While this is not an issue in our evaluation dataset, we consider this necessary for an operational deployment. One of the interesting aspects of our semantics-aware approach is that it results in signatures with semantic context that are easily parsed. Misconfiguration could likely be separated by picking from clusters with a large number of sources sent to a large number of destinations ${ }^{5}$. However, fully-automating $\mathrm{Ne}$ mean and making it immune to data pollution remains an area of future work.
One reason for this requirement is that unlike systems such as EarlyBird and Autograph, the target of attacks we seek to address is much broader than flash worms. It includes everyday targeted attacks, viruses spreading through network shares and botnet sweeps that occur below the noise thresholds and look similar to misconfiguration. We expect intentional data pollution through large botnets to be an issue for aforementioned systems as well.

\section{DAC Implementation}

We have implemented prototypes of each Nemean component. While the Nemean design provides flexibility to handle any protocol, we focus our discussion on two specific protocol implementations, HTTP (port 80) and NetBIOS/SMB (ports 139 and 445), since these two services exhibit great diversity in the number and types of exploits.

- Transport-Level Normalization: Transport-level normalization resolves ambiguities introduced at the network (IP) and transport (TCP) layers of the protocol stack. We check message integrity, reorder packets as needed, and discard invalid or duplicate packets. The importance of transport layer normalizers has been addressed in the literature [6,21]. Building a normalizer that perfectly resolves all ambiguities is a complicated endeavor, especially since many ambiguities are operating system dependent. We can constrain the set of normalization functions for two reasons. First, we only consider traffic sent to honeynets, so we have perfect knowledge of the host environment. This environment remains relatively constant. We do not need to worry about ambiguities introduced due to DHCP or network address translation (NAT). Second, Nemean's current implementation analyzes network traces off-line which relaxes its state holding requirements and makes it less vulnerable to resource-consumption attacks.

Attacks that attempt to evade a NIDS by introducing ambiguities to IP packets are well known. Examples of such attacks include simple insertion attacks that would be dropped by real systems but are evaluated by NIDS, and evasion attacks that are the reverse [21]. Since Nemean obtains traffic promiscuously via a packet sniffer (just like real a NIDS), these ambiguities must be resolved. We focus on three common techniques used by attackers to elude detection.

First, an invalid field in a protocol header may cause a NIDS to handle the packet differently than the destination machine. Handling invalid protocol fields in IP packets involves two steps: recognizing the presence of the invalid fields and understanding how a particular operating system would handle them. Our implementation performs some of these validations. For example, we 
1. Build the multiset $C$ of all normalized connections.

2. Cluster $C$ into exclusive partitions $\mathcal{C} \mathcal{L}=\left\{\xi_{i}\right\}$.

3. Produce a connection-level signature $\phi_{\xi}$ for each cluster by generalizing cluster data.

4. Build the multiset $S^{\prime}$ of all sessions. Each session $s^{\prime} \in S^{\prime}$ is a sequence of identifiers denoting the connection clusters that contain each connection in the session.

5. Cluster $S^{\prime}$ into partitions $\Psi=\left\{\psi_{i}\right\}$.

6. Produce a session-level signature $L_{\psi}$ for each cluster, generalizing the observed connection orderings.

7. Produce a NIDS signature. The signature is a hierarchical automaton where each transition in the session-level signature requires that the connection-level signature for the identified connection cluster accepts.

Figure 3: Multi-level Signature Generalization (MSG) algorithm. Section 5 provides more complete details.

drop packets with an invalid IP checksum or length field.

Second, an attacker can use IP fragmentation to present different data to the NIDS than to the destination. Fragmentation introduces two problems: correctly reordering shuffled packets and resolving overlapping segments. Various operating systems address these problems in different ways. We adopt the alwaysfavor-old-data method used by Microsoft Windows. A live deployment must either periodically perform activemapping [26] or match rules with passive operating system fingerprinting. The same logic applies for fragmented or overlapping TCP segments.

Third, incorrect understanding of the TCP Control Block (TCB) tear-down timer can cause a NIDS to improperly maintain state. If it closes a connection too early it will lose state. Likewise, retaining connections too long can prevent detection of legitimate later connections. Our implementation maintains connection state for an hour after session has been closed. However, sessions that have been closed or reset are replaced earlier if a new connection setup is observed between the same host/port pairs.

- Service-Level Normalization: We provide a brief discussion of the implementation of service normalizers for two popular protocols: HTTP and NetBIOS/SMB.

Ambiguities in HTTP sessions are primarily introduced due to invalid protocol parsing or invalid decoding of protocol fields. In particular, improper URL decoding is a point of vulnerability in many intrusion detection systems. Modern web servers allow substitution of encoded characters for ASCII characters in the URL and are often exploited as means for evasion of common NIDS signatures. Our DAC correctly decodes several observed encodings such as hex encoding and its variants, UTF-8 encoding, bare-byte encoding, and Microsoft Unicode encoding. Regardless of its encoding, the DAC presents a canonical URL in ASCII format to the clustering module. Currently, our implementation does not handle all obvious HTTP obfuscations. For example, we do not process pipelined HTTP/1.1 requests. Such requests need to be broken into multiple connec- tions for analysis. We plan to incorporate this functionality into our system in the future.

NetBIOS is a session-layer service that enables machines to exchange messages using names rather than IP addresses and port numbers. SMB (Server Message Block) is a transport-independent protocol that provides file and directory services. Microsoft Windows machines use NetBIOS to exchange SMB file requests. NetBIOS/SMB signature evasion techniques have not been well studied, possibly due to the lack of good NIDS rules for their detection. A full treatment of possible NetBIOS/SMB ambiguities exceeds the scope of this paper.

\section{Multi-level Signature Generalization}

We designed the Multi-level Signature Generalization $(M S G)$ algorithm to automatically produce signatures for normalized session data. The signatures must balance specificity to the exploits observed in the data with generality, the ability to detect attack variants not previously observed. We use machine-learning algorithms, including clustering and finite state machine generalization, to produce signatures that are well-balanced.

Due to the hierarchical nature of the session data, we construct signatures for connections and sessions separately. First, we cluster all connections irrespective of the sessions that contain them and generalize each cluster to produce a signature for each connection cluster. Second, we cluster sessions based upon their constituent connections and then generalize the clusters. Finally, we combine the session and connection signatures to produce a hierarchical automaton signature, where each connection in a session signature must match the corresponding connection signature. Figure 3 presents a high-level overview of the algorithm.

Steps 1 and 2: Generating connection clusters. Let $S$ be the multiset of normalized sessions produced by the data abstraction component. Denote each session $s \in S$ as an ordered list of connections: $s=c_{1} \cdot c_{2} \cdot \cdots \cdot c_{n_{s}}$. Let $\operatorname{Conn}(s)=\left\{c_{i}\right\}_{i=1 \ldots n_{s}}$ be the multiset of connections in $s$ and $C=\biguplus_{s \in S} \operatorname{Conn}(s)$ be the multiset of all con- 
nections in the normalized data, where $\uplus$ denotes multiset union. Let $\mathcal{C} \mathcal{L}=\left\{\xi_{i}\right\}_{i=1 \ldots m}$ be an exclusive clustering of $C$ into $m$ clusters $\xi_{i}$. Clustering inserts every element into a partition, so $\biguplus_{i=1}^{m} \xi_{i}=C$. Exclusive clustering requires that no partitions overlap, so $\xi_{i} \cap \xi_{j}=\emptyset$ for $i \neq j$. It immediately follows that there exists a welldefined function $\Gamma: C \rightarrow \mathcal{C L}$ defined as $\Gamma(c)=\xi$ if $c \in \xi$ that returns the cluster containing $c$. Section 5.1 presents the implementation of the clustering algorithm.

Step 3: Building connection-level signatures. Learning algorithms generalize the data in each cluster to produce signatures that match previously unseen connections. Let $\Sigma$ be the alphabet of network events comprising connection data. A learning algorithm is a function Learn $: \mathcal{P}\left(\Sigma^{*}\right) \rightarrow \mathcal{P}\left(\Sigma^{*}\right)$ that takes a set of strings $\widehat{\phi_{\xi}}=\bigcup_{c \in \xi} c$ and returns a regular language $\phi_{\xi} \supseteq \widehat{\phi_{\xi}}$. Section 5.2 presents the generalization algorithms used in our work. We recognize $\phi_{\xi}$ with a regular automaton that is the connection-level signature for cluster $\xi$.

Steps 4 and 5: Generating session clusters. Rewrite the existing sessions to produce a new set $S^{\prime}$.

$$
S^{\prime}=\biguplus_{s=c_{1} \cdots \cdot c_{n_{s}} \in S}\left[s^{\prime}=\Gamma\left(c_{1}\right) . \cdots . \Gamma\left(c_{n_{s}}\right)\right]
$$

From an implementation perspective, each $\Gamma\left(c_{i}\right)$ in a rewritten session is simply an integer index indicating which connection cluster contains the original connection. Intuitively, we allow any connection $c_{i}$ comprising part of session $s$ to be replaced with any connection $c_{i}^{\prime} \in \Gamma\left(c_{1}\right)$ identified by clustering as similar. Let $\Psi$ be a clustering of $S^{\prime}$.

Steps 6 and 7: Building session-level signatures. As with connection-level generalization, construct a regular language $L_{\psi}$ for each cluster $\psi \in \Psi$ that accepts the sessions in $\psi$ and variants of those sessions. Again, we recognize the language with a finite automaton. The connection cluster identifiers $\Gamma(c)$ label transitions in the session-level automata. The resulting signature is thus hierarchical: traversing a transition in the session signature requires connection data matching the signature for the connection cluster.

\subsection{Star Clustering Implementation}

We cluster connections and sessions using the same algorithm. We implemented the on-line star clustering algorithm, which clusters documents based upon a similarity metric [2]. This algorithm has advantages over more commonly-known techniques, such as the $k$-means family of algorithms [14]. For example, star clustering is robust to data ordering. Conversely, $k$-means produces different clusters depending upon the order in which data is read. Moreover, we need not know a priori how many

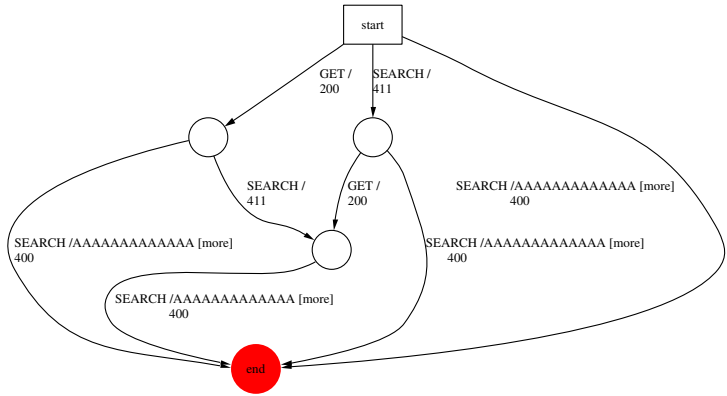

Figure 4: Welchia session level signature. For brevity, we label a single transition with both a request and a reply.

clusters are expected. Although it seems suitable, we make no claims that star is the optimal clustering algorithm for our purposes, and we expect to consider other algorithms in future work.

Star clustering builds a star cover over a partiallyconnected graph. Nodes in the graph each represent one or more items with semantically equivalent data. We arbitrarily choose one item at each node to be the representative item. A link exists between two nodes if the similarity between the corresponding representative items is above a designated threshold. A star cluster is a collection of nodes in the graph such that each node connects to the cluster center node with an edge. A star cover is a collection of star clusters covering the graph so that no two cluster centers have a connecting edge. In the original algorithm, a non-center node may have edges to multiple center nodes and thus appear in multiple clusters. We implemented a modified algorithm that inserts a node only into the cluster with which it has strongest similarity to produce an exclusive clustering.

Item similarity determines how edges are placed in the graph. We implemented two different similarity metrics to test sensitivity: cosine similarity [2] and hierarchical edit distance. The cosine similarity metric has lower computational complexity than hierarchical edit distance and was used for our experiments in Section 7.

Cosine similarity computes the angle between two vectors representing the two items under comparison. For each connection $A$, we build a vector $D_{A}$ giving the distribution of bytes, request types, and response codes that appeared in the network data. For sessions, the vector contains the distribution of connection cluster identifiers. If $\theta$ is the angle between vectors $D_{A}$ and $D_{B}$ representing items $A$ and $B$, then:

$$
\cos \theta=\frac{D_{A} \cdot D_{B}}{\left\|D_{A}\right\|\left\|D_{B}\right\|}
$$

where '.' represents inner product and $\|v\|$ is the vector 


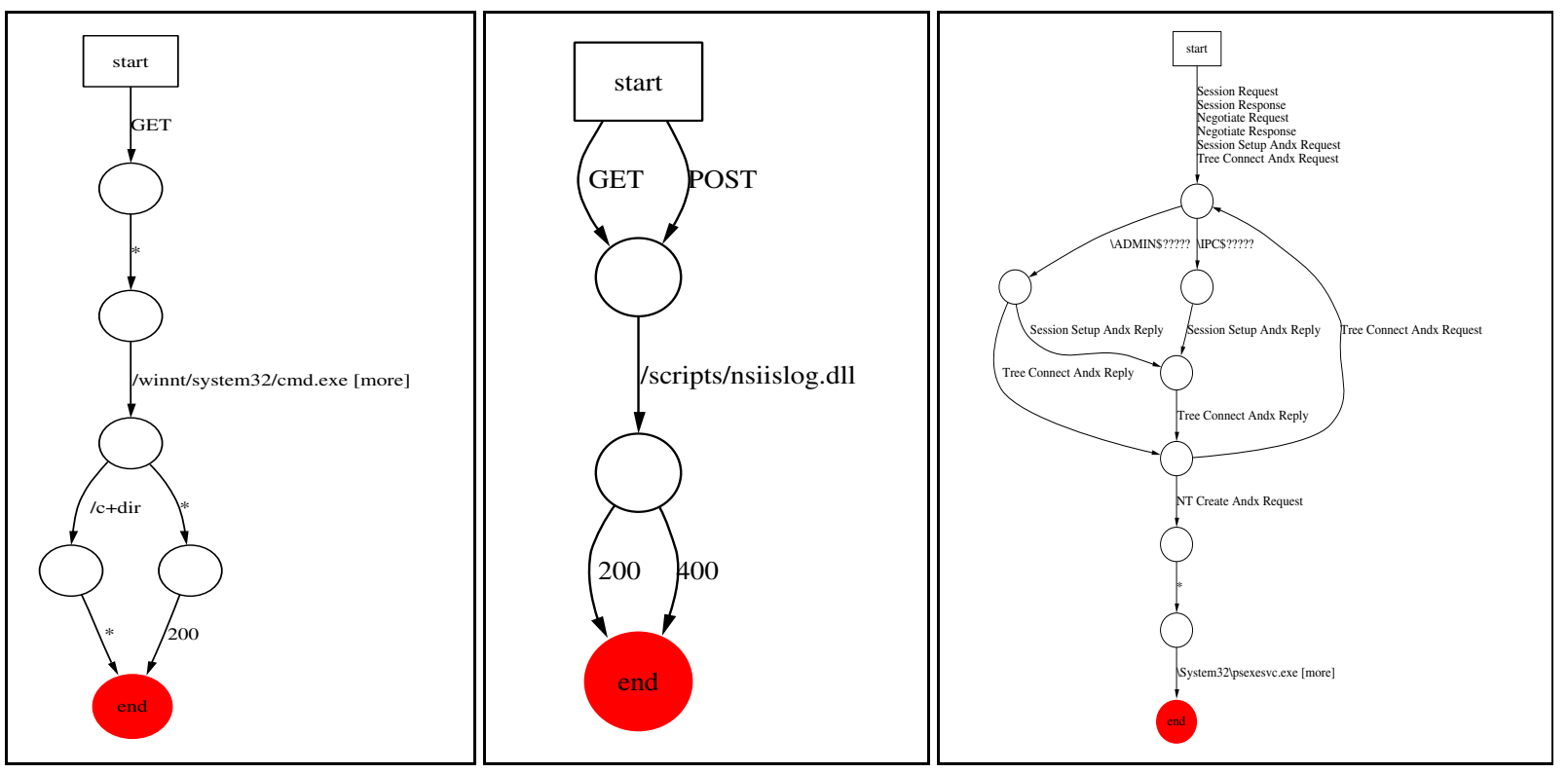

Figure 5: Nimda, Windows Media Player Exploit, and Deloder connection level signatures. The "*” transitions in the Nimda signature match any $\sigma \in \Sigma^{*}$.

norm. All vector values are non-negative, so $0 \leq \theta \leq$ $\pi / 2$ and $1 \geq \cos \theta \geq 0$. The similarity between items is the value $\cos \theta$, with $\cos \theta=1$ indicating equality.

Hierarchical-edit distance is a variation on the traditional edit-distance metric [2] which measures the cost of transforming one string into another using insert, delete, and replace operations. In contrast to the traditional editdistance metric, the hierarchical-edit distance metric preserves connection ordering information within each session and differentiates between the various data fields within each connection. We believed these properties of the hierarchical-edit distance metric would make it a better similarity metric for clustering than the cosine metric. Our experiments revealed that while both distance metrics work quite well, cosine is less sensitive to the threshold parameters used in partitioning clusters. Hence, we use cosine distance in this paper's experiments and describe the hierarchical edit distance metric in the expanded technical report [35].

Using a similarity metric, we construct the partiallyconnected similarity graph. An edge connects a pair of nodes if the similarity of the representative sessions is above a threshold, here 0.8 . We then build a star cover over the similarity graph. Each star cluster is a group of similar sessions that presumably are variants of the same exploit. The cluster set is then passed to the generalization module to produce the automaton signature.

\subsection{Cluster Generalization and Signature Generation}

Signature generation devises a NIDS signature from a cluster of similar connections or sessions. We generalize variations observed in a cluster's data. Assuming effective clustering, these variations correspond to obfuscation attempts or differences among variants of the same attack. By generalizing the differences, we produce a resilient signature that accepts data not necessarily observed during the training period.

The signature is a finite state automaton. We first construct a probabilistic finite state automaton (PFSA) accepting exactly the event sequences contained in a cluster, with edge weights corresponding to the number of times an edge is traversed when accepting all cluster data exactly once. PFSA learning algorithms [24] then use stochastic measures to generalize the data variations observed in a cluster. In this work, we generalized HTTP connection-level signatures with the $s k$-strings method [24], an algorithm that merges states when they are probabilistically indistinguishable. Session-level clusters were generalized with beam search [17]. Our algorithm uses both sk-strings and simulated beam annealing [23] to generalize NetBIOS signatures. These generalizations add transitions into the state machine to accommodate such variations as data reordering and alteration of characters in an attack string. Likewise, repeated strings may be generalized to allow any number of repeats.

We further generalize signatures at points of high data 
variability. Subsequence creation converts a signature that matches a sequence of session data into a signature that matches a subsequence of that data by inserting "gaps" that accept any sequence of arbitrary symbols. We insert gaps whenever observing four or more patterns with a common prefix, common suffix, and one dissimilar data element. For example, let $A, B \in \Sigma^{*}$ and $v, w, x, y \in \Sigma$. If the signature accepts $A v B, A w B$, $A x B$, and $A y B$, then we replace those four sequences with the regular expression $A[*] B$. Intuitively, we have identified a portion of the signature exhibiting large variation and allow it vary arbitrarily in our final signature. Nemean's generalized signatures can thus detect variations of observed attacks.

Figure 4 shows a session-level signature for Welchia, a worm that exploits a buffer overflow. Nemean's generalization produced a signature that matches a wide class of Welchia scans without losing the essential buffer overflow information characteristic to the worm. Figure 5 shows connection-level signatures for Nimda, a Windows Media Player exploit, and the Deloder NetBIOS worm. The connection-level Nimda signature is an example of a signature for an exploit with high diversity. In particular, note that the subsequence creation generalization allows this signature to match a wide class of Nimda attacks. The Windows Media Player exploit is representative of an HTTP exploit where the size of the exploit URL is small. Previous signature generation techniques, such as Honeycomb, fail for small URLs. The Deloder signature demonstrates the capability of Nemean to generate signatures for exploits using more complex protocols like NetBIOS/SMB.

\section{Data Collection}

The data used for our evaluation comes from two sources: (i) honeypot packet traces collected from unused address space that we used to build signatures and evaluate the detection capability of Nemean and (ii) packet traces collected from our departmental border router that we used to test the resilience of our signatures to false positives.

- Production Traffic: Obtaining packet traces for live network traffic is a challenge due to privacy concerns. While network operators are amenable to sharing flow level summaries, anonymizing payloads remains an unsolvable problem and as such its hard to obtain packet traces with application payloads.

We were able to obtain access to such data from our department's border router. The network is a sparsely allocated, well managed /16 network with approximately 24 web servers and around 400 clients. We were able to passively monitor all outgoing and incoming HTTP packets on this network for an 8 hour period. Table 1 provides a summary of this dataset.

- Honeypot Traffic: Traffic from two unused /19 IP address blocks totaling $16 \mathrm{~K}$ addresses from address ranges allocated to our university was routed to our honeynet monitoring environment. To normalize the traffic received by our infrastructure a simple sourcefiltering rule was employed: one destination IP address per source. Connections to additional destination IP addresses were dropped by the filter.

These filtered packets were subsequently routed to one of two systems based upon type-of-service. HTTP requests were forwarded to a fully patched Windows 2000 Server running on VMware. The NetBIOS/SMB traffic was routed to a virtual honeypot system similar to Honeyd. We routed NetBIOS/SMB packets to an active responder masquerading as an end host offering NetBIOS services rather than to the Windows 2000 Server for two reasons [33]. First, the fully patched Windows 2000 Server often rejected or disconnected the session before we had enough information to classify the attack vector accurately. This could be due to invalid NetBIOS names or user/password combinations. Our active responder accepted all NetBIOS names and user/password combinations. Second, Windows 2000 servers limit the number of simultaneous network share accesses which also inhibit connection requests from succeeding.

We collected two sets of traces, a short term training set ( 2 days) and a longer testing set ( 7 days) to evaluate Nemean detection capability as summarized in Table 2. The reduction in the volume of port 80 traffic moving from the 2-day to the 5-day dataset is not uncommon in honeynets due to the bursty nature of this traffic often associated with botnet activity [16].

\section{Evaluation}

We tested the effectiveness of Nemean's HTTP and NetBIOS signatures and examined the session clusters used to produce these signatures. Section 7.1 reveals the major classes of attacks in our recorded data and quantitatively measures the clusters produced by the clustering module. We performed an evaluation of the detection and false positive rates of Nemean's signatures and compare our results with Snort's HTTP capabilities. Finally, we provide a qualitative discussion of our experience with Honeycomb.

\subsection{Evaluating the Clusters}

- HTTP Clusters: Figure 6 provides an overview of the major HTTP clusters in our learning data set. WebDAV scans account for the majority of the attacks in our data set. WebDAV is a collection of HTTP extensions that allow users to collaboratively edit and man- 


\begin{tabular}{|c|r|r|r|r|}
\hline Data Flow & No. Clients & No. Servers & No. Sessions & No. Connections \\
\hline Internal clients - > External servers & 380 & 4,422 & 16,826 & 106,456 \\
\hline External clients - > Internal servers & 18,634 & 24 & 28,491 & 87,545 \\
\hline
\end{tabular}

Table 1: Production data summary (HTTP: 8 hours, 16GB).

\begin{tabular}{|c|r|r|r|r||r|r|r|r|}
\hline & \multicolumn{4}{|c||}{ Learning Data (2 days) } & \multicolumn{4}{c|}{ Test data (7 days) } \\
\hline Port & Packets & Sources & Connections & Sessions & Packets & Sources & Connections & Sessions \\
\hline $\mathbf{8 0}$ & 278,218 & 10,859 & 25,587 & 12,545 & 100,291 & 12,925 & 12,903 & 5,172 \\
\hline $\mathbf{1 3 9}$ & 192,192 & 1,434 & 3,415 & 1,657 & $6,764,876$ & 539,334 & $1,662,571$ & 24,747 \\
\hline $\mathbf{4 4 5}$ & $1,763,276$ & 14,974 & 35,307 & 19,763 & $6,661,276$ & 383,358 & $1,171,309$ & 37,165 \\
\hline
\end{tabular}

Table 2: Honeypot data summary.

age documents in remote web servers. Popular WebDAV methods used in exploits include OPTIONS, SEARCH, and PROPFIND and are supported by Microsoft IIS web servers. Scans for exploits of WebDAV vulnerabilities are gaining in popularity and are also used by worms like Welchia. Nimda attacks provide great diversity in the number of attack variants and HTTP URL obfuscation techniques. These attacks exploit directory traversal vulnerabilities on IIS servers to access $\mathrm{cmd}$. exe or root . exe. Figure 5 contains a connection-level signature for Nimda generated by Nemean. Details of other observed exploits, such as Frontpage, web crawlers and open-proxy, are provided in [35].

- NetBIOS Clusters: Worms that are typically better known as email viruses dominate the NetBIOS clusters. Many of these viruses scan for open network shares and this behavior dominated the observed traffic. They can be broadly classified into three types:

1. Hidden and open share exploits: This includes viruses, including LovGate [5], NAVSVC, and Deloder [12], that use brute force password attacks to look for open folders and then deposit virus binaries in startup folders.

2. MS-RPC query exploits: Microsoft Windows provides the ability to remotely access MSRPC services through named pipes such as epmapper (RPC Endpoint Mapper), srvsvc (Windows Server Service), and samr (Windows Security Account Manager). Viruses often connect to the MSRPC services as guest users and then proceed to query the system for additional information that could lead to privileged user access. For example, connecting to the samr service allows the attacker to obtain an enumeration of domain users,

3. MS-RPC service buffer overflow exploits: The most well-known of these exploits are the epmapper service which allows access to the RPC-DCOM exploit [15] used by Blaster and the more recent 1sarpc exploit used by Sasser [25]. We provide more details in the technical report [35].

- Cluster Quality: We quantitatively evaluated the quality of clusters produced by the star clustering algorithm using two common metrics: precision and recall. Precision is the proportion of positive matches among all the elements in each cluster. Recall is the fraction of positive matches in the cluster among all possible positive matches in the data set. Intuitively, precision measures the relevance of each cluster, while recall penalizes redundant clusters.

We first manually tagged each session with conjectures as shown in Figure 6. Conjectures identified sessions with known attack types and it is possible for a session to be marked with multiple conjectures. It is important to note that these conjectures were not used in clustering and served simply as evaluation aids to estimate the quality of our clusters.

The conjectures allow us to compute weighted precision (wp) and weighted recall (wr) for our clustering. As sessions can be tagged with multiple conjectures, we weight the measurements based upon the total number of conjectures at a given cluster of sessions. We compute the values $w p$ and $w r$ as follows: Let $C$ be the set of all clusters, $J$ be the set of all possible conjectures, and $c_{j}$ be the set of elements in cluster $c$ labeled with conjecture $j$. Then $\left|c_{j}\right|$ is the count of the number of elements in cluster $c$ with conjecture $j$.

$$
\begin{aligned}
w p & =\sum_{c \in C}\left(\frac{|c|}{|C|} \sum_{j \in J}\left(\frac{\left|c_{j}\right|}{\sum_{k \in J}\left|c_{k}\right|} \frac{\left|c_{j}\right|}{|c|}\right)\right) \\
& =\frac{1}{|C|} \sum_{c \in C} \frac{\sum_{j \in J}\left|c_{j}\right|^{2}}{\sum_{k \in J}\left|c_{k}\right|} \\
w r & =\sum_{c \in C}\left(\frac{|c|}{|C|} \sum_{j \in J}\left(\frac{\left|c_{j}\right|}{\sum_{k \in J}\left|c_{k}\right|} \frac{\left|c_{j}\right|}{\left|C_{j}\right|}\right)\right) \\
& =\frac{1}{|C|} \sum_{c \in C}\left(\frac{|c|}{\sum_{k \in J}\left|c_{k}\right|} \sum_{j \in J} \frac{\left|c_{j}\right|^{2}}{\left|C_{j}\right|}\right)
\end{aligned}
$$




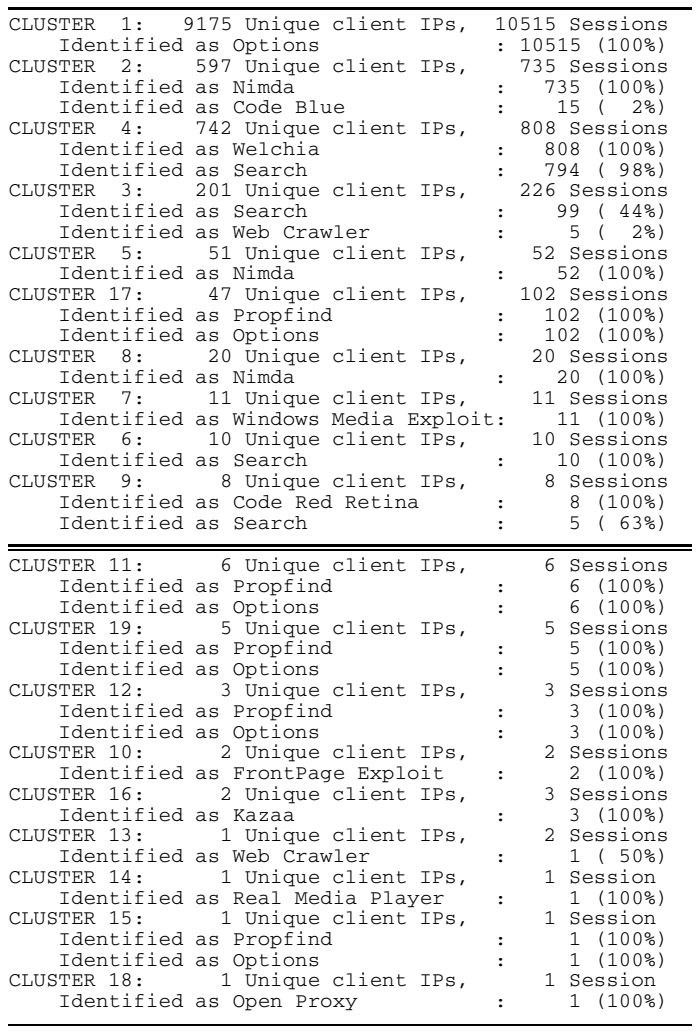

Figure 6: HTTP Port 80 cluster report.

In the formulas above, $\sum_{k \in J}\left|c_{k}\right| \geq|c|$ and $\sum_{k \in J}\left|C_{k}\right| \geq|C|$ as sessions may have multiple conjectures. Figure 7 presents graphs indicating how precision and recall vary with the clustering similarity threshold. Recall that in the star clustering algorithm, an edge is added between two sessions in the graph of all sessions only if their similarity is above the threshold. Although less true for NetBIOS data, the similarity threshold does not have a significant impact on the quality of the resulting clustering. Clustering precision drops as the threshold nears 0 because the star graph becomes nearly fully connected and the algorithm cannot select suitable cluster centers. Recall that no cluster centers can share an edge, so many different clusters merge together at low threshold values. At the clustering threshold used in our experiments (0.8), precision scores were perfect or nearly perfect.

\subsection{Signature Effectiveness}

Intrusion detection signatures should satisfy two basic properties. First, they should have a high detection rate; i.e., they should not miss real attacks. Second, they should generate few false alarms. Our results will show that Nemean has a $99.9 \%$ detection rate with 0 false alarms. Two additional metrics evaluate the quality of the alarms raised by an IDS. Precision empirically evaluates alarms by their specificity to the attack producing the alarm. Noise level counts the number of alarms per incident and penalizes redundant alarms. In these tests, we use Snort as a baseline for comparison simply because that is the most widely adopted intrusion detection system. We used the latest version of Snort available at the time, Snort-2.1.0 with the HTTP pre-processor enabled, and its complete associated ruleset. In some sense, Snort is the strawman because of its well-known susceptibility to false-positives. We use this because of our inability to compare with Honeycomb (see Section 7.4) and because there is no source code publicly available for Earlybird or Autograph [10,27].

- 99.9\% Detection Rate: We evaluated the detection rate of Nemean signatures using leave-out testing, a common technique in machine learning. We used the honeynet data set described in Table 2 to automatically create connection-level and session-level signatures for the clusters identified in a training data set. We measured the detection rate of the signatures by running signature matching against data in a different trace collected from the same network (see Table 2).

Connection-level HTTP signatures detected $100.0 \%$ of the attacks present, and the somewhat more restrictive session-level signatures detected $97.7 \%$. We did not evaluate session-level signatures for Nimda because the extreme variability of Nimda attacks made such signatures inappropriate. Table 3 shows the number of occurrences of the HTTP attacks and the number detected by Nemean signatures. For comparison, we provide detection counts for Snort running with an up-to-date signature set. Snort detected $99.7 \%$ of the attacks.

The detection rate of NetBIOS attacks is similarly very high: we detected $100.0 \%$ of the attacks present. Table 4 contains the detection rates for NetBIOS/SMB signatures. Snort provides only limited detection capability for NetBIOS attacks, so a comparison was infeasible. All signatures were connection-level because the defining characteristic of each attack is a string contained in a single connection. The structure of connections within a session is irrelevant for such attacks.

- Zero misdiagnoses or false alarms: We qualify incorrect alerts on the honeynet data as misdiagnoses. Although not shown in Table 3, all Nemean HTTP signatures generated 0 misdiagnoses on the honeynet trace. Misdiagnosis counts for NetBIOS/SMB on the honeynet data were also 0, as shown in Table 4 . We also measured false alarm counts of Nemean HTTP signatures against 16GB of packet-level traces collected from our department's border router over an 8 hour time period. The traces contained both inbound and outbound HTTP traffic. We evaluated both Nemean and Snort against the dataset. 

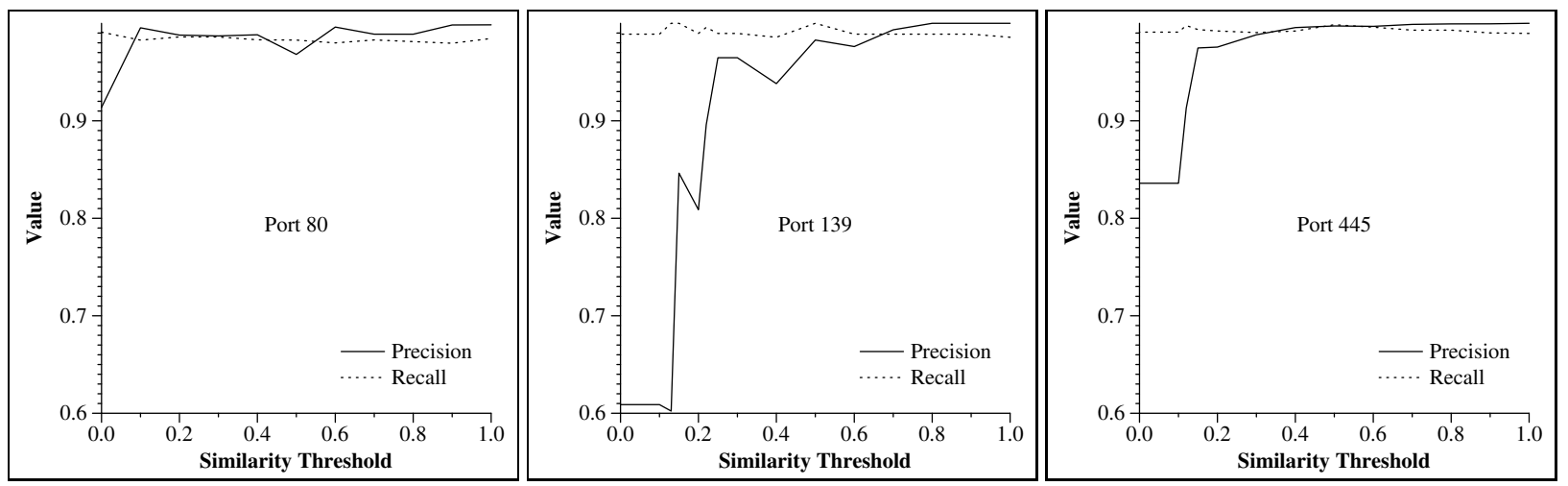

Figure 7: Effect of clustering similarity threshold upon weighted precision and weighted recall. Note that the y-axis begins at 0.6 .

\begin{tabular}{|l|r||r|r|r|}
\hline \multirow{2}{*}{ Signature } & \multicolumn{1}{|c||}{} & \multicolumn{2}{c|}{ Nemean } & \multirow{2}{*}{ Present } \\
\cline { 3 - 4 } & 1172 & 1172 & 1160 & 1171 \\
\hline Options & 496 & 496 & N/A & 495 \\
\hline Nimda & 229 & 229 & 205 & 229 \\
\hline Propfind & 90 & 90 & 90 & 90 \\
\hline Welchia & 89 & 89 & 89 & 89 \\
\hline Win Media Player & 4 & 4 & 4 & 0 \\
\hline Code Red Retina & 2 & 2 & 2 & 2 \\
\hline Kazaa & & & &
\end{tabular}

\begin{tabular}{|l|r|}
\hline \multicolumn{1}{|c|}{ Signature } & No. Alerts \\
\hline Non-RFC HTTP Delimiter & 32246 \\
\hline Bare Byte Unicode Encoding & 28012 \\
\hline Apache Whitespace (TAB) & 9950 \\
\hline WEB-MISC /doc/ Access & 9121 \\
\hline Non-RFC Defined Character & 857 \\
\hline Double-Decoding Attack & 365 \\
\hline IIS Unicode Codepoint Encoding & 351 \\
\hline
\end{tabular}

Table 3: Session-level HTTP signature detection counts for Nemean signatures and Snort Signatures. We show only exploits occurring at least once in the training and test data.

\begin{tabular}{|l|r||r|r|}
\hline Signature & Present & Detected & Misdiagnoses \\
\hline Srvsvc & 19934 & 19930 & 0 \\
\hline Samr & 8743 & 8741 & 0 \\
\hline Epmapper & 1263 & 1258 & 0 \\
\hline NvcplDmn & 62 & 61 & 0 \\
\hline Deloder & 30 & 30 & 0 \\
\hline LoveGate & 1 & 0 & 0 \\
\hline
\end{tabular}

Table 4: Detection and misdiagnosis counts for connection-level Nemean NetBIOS signatures. This data includes both port 139 and port 445 traffic.

Nemean results are highly encouraging: 0 false alarms. Snort generated 88,000 alarms on this dataset, almost all of which were false alarms. The Snort false alarms were produced by a collection of overly general signatures. In fairness, we note that Snort had a larger signature set which made it more prone to false positives. Our Snort signature set included about 2200 signatures, whereas Nemean's database of HTTP and NetBIOS signatures contained only 22 connection-level and 7 session-level signatures. Snort has a high signature count because it is meant to detect classes of attacks be-

Table 5: Snort false alarm summary for over 45,000 HTTP sessions collected from our department's border router.

\begin{tabular}{|l|r|r|}
\hline \multicolumn{1}{|c|}{ Alert Category } & No. Signatures & No. Alerts \\
\hline WEB-MISC & 13 & 466 \\
\hline WEB-CGI & 25 & 919 \\
\hline WEB-IIS & 8 & 164 \\
\hline WEB-ATTACKS & 6 & 15 \\
\hline WEB-PHP & 4 & 18 \\
\hline WEB-FRONTPAGE & 4 & 61 \\
\hline Others (P2P, Crawlers) & 5 & 5426 \\
\hline
\end{tabular}

Table 6: Summary of remaining Snort alerts.

yond those seen by Honeynets.

Table 5 provides a summary of the Snort alarms generated on an 8 hour trace of overwhelmingly benign HTTP traffic collected at our department's border router. Reducing Snort's alarm rate would require reengineering of many signatures. Additionally, the overly general signature provides little specific information about the type of exploit that may be occurring.

We assume that in a real network deployment of Snort the most noisy signatures such as those in Table 5 would be disabled. A more reasonable estimate of the expected false alarm rates might be obtained from the remaining alerts shown in Table 6. The remaining alerts come from 60 signatures and are responsible for 1643 alerts (exclud- 
ing Others). While we did not inspect each of these individually for true positives due to privacy concerns with the dataset, sampling revealed that they are mostly false alarms. Traffic classified as Others were legitimate alerts fired on benign P2P traffic and traffic from web crawlers.

Our university filters NetBIOS traffic at the campus border, so we were unable to obtain NetBIOS data for this experiment.

- Highly specific alarms: Although the decision is ultimately subjective, we believe our signatures generate alerts that are empirically better than alerts produced by packet-level systems such as Snort. Typical Snort alerts, such as "Bare Byte Unicode Encoding" and "Non-RFC HTTP Delimiter", are not highly revealing. They report the underlying symptom that triggered an alert but not the high-level reason that the symptom was present. This is particularly a problem for NetBIOS alerts because all popular worms and viruses generate virtually the same set of alerts. We call these weak alerts and describe them in more detail in the technical report [35]. Nemean, via connection-level or session-level signatures, has a larger perspective of a host's intentions. As a result, we generate alerts specific to particular worms or known exploits.

- Low noise due to session-level signatures: Moreover, Nemean provides better control over the level of noise in its alarms. Packet-level detection systems such as Snort often raise alerts for each of multiple packets comprising an attack. A security administrator will see a flurry of alerts all corresponding to the same incident. For example, a Nimda attack containing an encoded URL will generate URL decoding alarms in Snort and alerts for WEB-IIS cmd. exe access. Sophisticated URL decoding attacks could later get misdiagnosed as Nimda alerts and be filtered by administrators. Our normalizer converts the URL to a canonical form to accurately detect Nimda attacks. Since Nemean aggregates information into connections or sessions and generates alerts only on the aggregated data, the number of alerts per incident is reduced.

In summation, we believe these results demonstrate the strength of Nemean. It achieves detection rates similar to Snort with dramatically fewer false alarms. The alerts produced by Nemean exhibit high quality, specifying the particular attack detected and keeping detection noise small.

\subsection{Signature Generation Efficiency}

Although our current implementation operates offline on collected data sets, we intend for Nemean to be used in online signature generation. Online systems must be efficient, both so that new signatures can be rapidly constructed as new attacks begin to appear and so that the system can operate at network speeds with low compu- tational demands. Figure 8 shows Nemean's overheads on the 2-day training data set containing about 200,000 HTTP packets and 2,000,000 NetBIOS packets. Total data processing time is divided into the three stages of data abstraction, clustering, and automaton generalization plus an additional preprocessing step that translated SSTs produced by the DAL into the input format of our clustering module. The HTTP connectionlevel automaton generalization step used the sk-strings algorithm. The session-level generalization used beam search, with nearly $100 \%$ of the cost arising from one cluster of Nimda sessions. At 200,000 packets, the cost of session-level generalization was 587 seconds. NetBIOS signature generalization used simulated beam annealing at the connection-level only, with no construction of session-level signatures.

Nemean is efficient. We are able to generate signatures for 2 days worth of NetBIOS data, totaling almost 2 million packets, in under 70 seconds. Even our most expensive operation, session-level generalization of HTTP data, required less than 10 minutes of computation. The design of our system helps keep costs low. By processing only data collected on a honeynet, the overall volume of data is significantly reduced. Deploying Nemean as an online signature generator would require limited system resources and can easily operate at the speeds of incoming data.

\subsection{Honeycomb Comparison}

Honeycomb was one of the first efforts to address the problem of automatic signature generation from honeypot traces. We performed a comparison between Nemean and Honeycomb on identical traces as a means to further understand the benefits of semantics awareness in automated signature generators. This evaluation was complicated by two issues: first, we transformed Honeycomb's Honeyd plug-in implementation into a standalone application by feeding it the input traffic from a pcap loop. Second, since Honeycomb was developed as a proof-ofconcept tool, it turned out to be incapable of processing large traces ${ }^{6}$. In our experience, Honeycomb's processing time grows quadratically with each connection since it performs pairwise comparison across all connections, and running it on a relatively small trace of 3000 packets took several hours on a high performance workstation. As a result, our evaluation is a qualitative comparison of Honeycomb signatures and its performance on a small trace with 126 HTTP connections.

Honeycomb produced 202 signatures from the input trace. While there were several perfectly functional signatures, there were also a surprisingly large number of benign strings that were identified by the LCS algorithm. Some of these were small strings such as "GET" or 

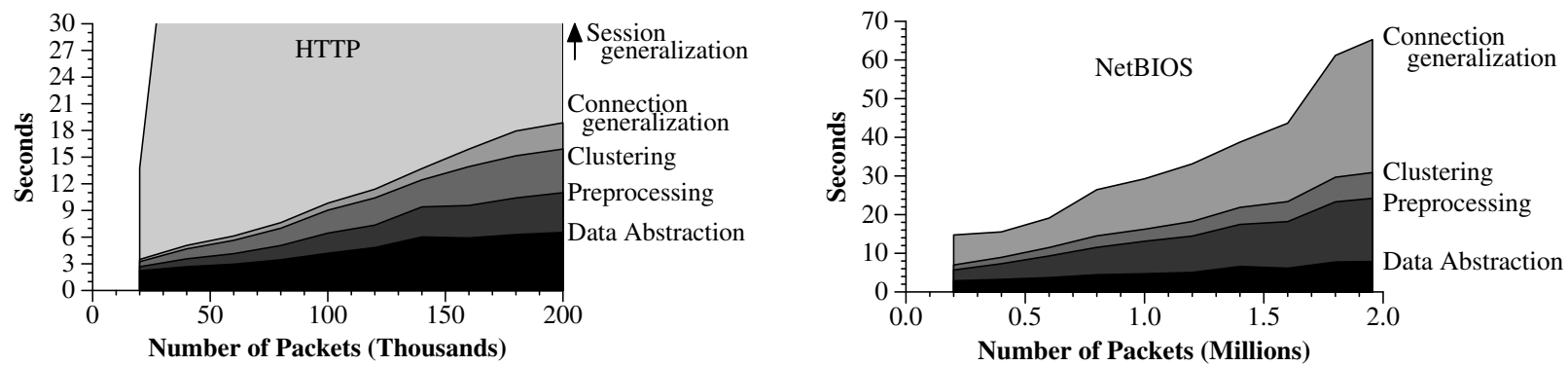

Figure 8: Time to construct signatures for HTTP and NetBIOS data, based upon the number of packets in the data set. Note that $\mathrm{X}$ and $\mathrm{Y}$ scales differ in the two graphs. Preprocessing is a data file translation step converting SSTs into the input format of our clustering module. HTTP session-level signature generalization required 587 seconds at 200,000 packets.

\begin{tabular}{|c|c|c|}
\hline Honeycomb Signature & Exploit & Deficiency \\
\hline 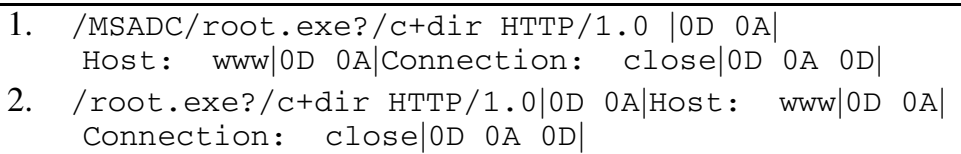 & Nimda & Redundant $^{7}$ \\
\hline 1. SEARCH / HTTP/1.1|0D 0A|Host: 128.1 & WebDAV & Restrictive $^{8}$ \\
\hline $\begin{array}{l}\text { 1. } \mid \text { OD 0A|Connection: Keep-Alive|0D OA OD| } \\
\text { 2. HTTP /1 }\end{array}$ & None & Benign \\
\hline
\end{tabular}

Table 7: Example signatures produced by Honeycomb on an HTTP trace with 126 connections.

"HTTP" that are clearly impractical and just happened to be the longest common substring between unrelated sessions. Communication with the Honeycomb author revealed these were part of normal operation and the typical way to suppress these are to whitelist signatures smaller than a certain length. There were also much longer strings in the signature set, such as proxy-headers that also do not represent real attack signatures. It seems that the only way to avoid these kinds of problems is through manual grooming of signatures by an expert with protocol knowledge. It should be noted that while Nemean also requires a sanity check process, this affects Honeycomb to a much greater extent because of it tendency to generate a large number of signatures.

The summary of the comparison of signatures produced by Honeycomb versus those produced by Nemean is as follows:

1. Honeycomb produces a large number of signatures that lack specificity due to pairwise connection comparison. Nemean's algorithm generalizes from a cluster that includes several connections resulting in a smaller, balanced signature set.

2. Pairwise LCS employed by Honeycomb often leads to redundant (non-identical) signatures, which would generate multiple alarms for the same attack. Again, Nemean's algorithm generalizes from clusters and its semantics awareness makes it far less prone to redundant signature production.
3. Honeycomb signatures are often too restrictive. As a result, we require several restrictive signatures to capture all instances of a particular attack and this could lead to false negatives. Nemean's generation of balanced signatures make them less susceptible to false negatives.

4. Honeycomb's lack of semantics awareness leads to signatures consisting of benign substrings. These lead to false positives and explains why Honeycomb is unable to produce precise signatures for protocols such as NetBIOS, MS-SQL and HTTP attacks, such as Nimda, where the exploit content is a small portion of the entire attack string. Nemean's semantics awareness addresses the issue of benign substrings.

We present examples of signatures that we obtained from Honeycomb that demonstrate these weaknesses in Table 7.

\section{Discussion}

A potential vulnerability of Nemean is its use of honeynets as a data source. If attackers become aware of this, they could either attempt to evade the monitor or to pollute it with irrelevant traffic resulting in many unnecessary signatures. Evasion can be complicated by periodic rotation of the monitored address space. Intentional 
pollution is a problem for any automated signature generation method and we intend to address it in future work.

Three issues may arise when deploying Nemean on a live network. First, live networks have real traffic, so we cannot assume that all observed sessions are malicious. To produce signatures from live traffic traces containing mixed malicious and normal traffic, we must first separate the normal traffic from the malicious. Flow-level anomaly detection or packet prevalence techniques [27] could help to identify anomalous flows in the complete traffic traces. Simple techniques that flag sources that horizontally sweep the address space, vertically scan several ports on a machine, and count the number of rejected connection attempts could also be used.

Second, Nemean must generate meaningful signatures for Snort, Bro, or other NIDS. Snort utilizes an HTTP preprocessor to detect HTTP attacks and does not provide support for regular expressions. Converting $\mathrm{Ne}$ mean signatures to Bro signatures is straightforward since Bro allows for creation of policy scripts that support regular expressions.

Third, while it is not the focus of the current implementation, the limited manual selection required suggests that automating deployment of Nemean signatures should be realizable. This resiliency of Nemean signatures to false positives makes it quite attractive as a means to automate defense against flash worms that propagate rapidly. The data abstraction component's modules work without any changes on live traces. The star clustering algorithm is also designed to perform incremental clustering and work in an online fashion. Anomaly detection techniques could be employed in parallel with Nemean to flag compelling clusters for worm outbreaks. Automatically generated Nemean signatures for these clusters could then be rapidly propagated to NIDS to defend against emergent worms.

\section{Conclusions}

We have described the design and implementation of Nemean, a system for automated generation of balanced NIDS signatures. One of the primary objectives of this system is to reduce false alarm rates by creating signatures that are semantics aware. Nemean's architecture is comprised of two major components: the data-abstraction component and the signature-generation component. This modular design supports and encourages independent enhancement of each piece of the architecture. Nemean uses packet traces collected at honeynets as input since they provide an unfettered view of a wide range of attack traffic.

We evaluated a prototype implementation of Nemean using data collected at two unused /19 subnets. We collected packet traces for two services for which we devel- oped service normalizers (HTTP and NetBIOS/SMB). Running Nemean over this data resulted in clusters for a wide variety of worms and other exploits. Our evaluation suggests that simple similarity metrics, such as the cosine metric, can provide clusters with a high degree of precision. We demonstrated the signature generation capability of our system and discussed optimizations used by our automata learning module, such as structure abstraction and subsequence creation. We showed that Nemean generated accurate signatures with extremely low false alarm rates for a wide range of attack types, including buffer overflows (Welchia), attacks with large diversity (Nimda), and attacks for complicated protocols like NetBIOS/SMB.

In future work, we intend to hone the on-line capabilities of Nemean and to assess its performance over longer periods of time in live deployments. We will also continue to evaluate methods for clustering and learning with the objective of fine tuning the resulting signature sets.

\section{Acknowledgements}

This work is supported in part by Army Research Office grant DAAD19-02-1-0304, Office of Naval Research grant N00014-01-1-0708, and National Science Foundation grant CNS-0347252. The first author was supported in part by a Lawrence H. Landweber NCR Graduate Fellowship in Distributed Systems. The second author was supported in part by a Cisco Systems Distinguished Graduate Fellowship.

The U.S. Government is authorized to reproduce and distribute reprints for Governmental purposes, notwithstanding any copyright notices affixed thereon. The views and conclusions contained herein are those of the authors and should not be interpreted as necessarily representing the official policies or endorsements, either expressed or implied, of the above government agencies or the U.S. Government.

We thank CSL for providing us access to the traces, Dave Plonka, Geoff Horne, Bill Jensen and Michael Hare for support of iSink project. Finally, we would like to thank Vern Paxson, our shepherd Fabian Monrose and the anonymous reviewers whose insightful comments have greatly improved the presentation of the paper.

\section{References}

[1] R. Agrawal, T. Imielinski, and A. Swami. Mining association rules between sets of items in large databases. In ACM SIGMOD International Conference on Management of Data, 1993.

[2] J. Aslam, K. Pelekhov, and D. Rus. A practical clustering algorithm for static and dynamic information organization. In $A C M$ SIAM Symposium on Discrete Algorithms (SODA), Baltimore, Maryland, January 1999. 
[3] B. Caswell and M. Roesch. The SNORT network intrusion detection system. http://www.snort.org, April 2004.

[4] M. Christodorescu, S. Seshia, S. Jha, D. Song, and R. E. Bryant. Semantics-aware malware detection. In IEEE Symposium on Security and Privacy, Oakland, California, May 2005.

[5] T. Conneff. W32.HLLW.lovgate removal tool. http://securityresponse.symantec.com/avcenter/venc/data/w32.hllw.lovgate.removal.tool.html, April 2004.

[6] M. Handley, V. Paxson, and C. Kreibich. Network intrusion detection: Evasion, traffic normalization and end-to-end protocol semantics. In $10^{\text {th }}$ USENIX Security Symposium, Washington, DC, August 2001.

[7] The Honeynet project. http://project.honeynet.org, April 2004.

[8] K. Julisch. Clustering intrusion detection alarms to support root cause analysis. ACM Transactions on Information and System Security (TISSEC), 6(4):443-471, November 2003.

[9] J. Jung, V. Paxson, A. W. Berger, and H. Balakrishnan. Fast portscan detection using sequential hypothesis testing. In IEEE Symposium on Security and Privacy, Oakland, California, May 2004.

[10] H.-A. Kim and B. Karp. Autograph: Toward automated, distributed worm signature detection. In $13^{\text {th }}$ USENIX Security Symposium, San Diego, California, August 2004.

[11] C. Kreibich and J. Crowcroft. Honeycomb-creating intrusion detection signatures using honeypots. In $2^{\text {nd }}$ Workshop on Hot Topics in Networks (Hotnets-II), Cambridge, Massachusetts, November 2003.

[12] K. Lai. Deloder worm/trojan analysis. http://www.klcconsulting.net/deloder_worm.htm, April 2004.

[13] J. Levine, R. LaBella, H. Owen, D. Contis, and B. Culver. The use of honeynets to detect exploited systems across large enterprise networks. In 2003 IEEE Workshop on Information Assurance, West Point, New York, June 2003.

[14] S. P. Lloyd. Least squares quantization in PCM. IEEE Transactions on Information Theory, IT-2:129-137, 1982.

[15] Microsoft security bulletin MS03-007. http://www.microsoft.com/technet/security/bulletin/MS03-007.asp, April 2004.

[16] R. Pang, V. Yegneswaran, P. Barford, V. Paxson, and L. Peterson. Characteristics of internet background radiation. In $A C M$ SIGCOMM/Usenix Internet Measurement Conference, 2004.

[17] J. Patrick, A. Raman, and P. Andreae. A Beam Search Algorithm for PFSA Inference, pages 121-129. Springer-Verlag London Ltd, $1^{\text {st }}$ edition, 1998.

[18] V. Paxson. BRO: A system for detecting network intruders in real time. In $7^{\text {th }}$ USENIX Security Symposium, San Antonio, Texas, January 1998.

[19] F. Pouget and M. Dacier. Honeypot-based forensics. In AusCERT Asia Pacific Information technology Security Conference 2004 (AusCERT2004), Brisbane, Australia, May 2004.

[20] N. Provos. A virtual honeypot framework. In USENIX Security Symposium, San Diego, CA, August 2004.

[21] T. Ptacek and T. Newsham. Insertion, evasion and denial of service: Eluding network intrusion detection. Technical report, Secure Networks, January 1998.

[22] R. F. Puppy. A look at Whisker's anti-IDS tactics. http://www.wiretrip.net/rfp/txt/whiskerids.html, April 2004

[23] A. Raman and J. Patrick. Beam search and simulated beam annealing. Technical Report 2/97, Department of Information Systems, Massey University, Palmerston North, New Zealand, 1997.
[24] A. V. Raman and J. D. Patrick. The sk-strings method for inferring PFSA. In $14^{\text {th }}$ International Conference on Machine Learning (ICML97), Nashville, Tennessee, July 1997.

[25] W32 Sasser.Worm. http://securityresponse.symantec.com/ avcenter/venc/data/w32.sasser.worm.html.

[26] U. Shankar and V. Paxson. Active mapping: Resisting NIDS evasion without altering traffic. In IEEE Symposium on Security and Privacy, Oakland, California, May 2003.

[27] S. Singh, C. Estan, G. Varghese, and S. Savage. Automated worm fingerprinting. In 6th Symposium on Operating Systems Design and Implementation (OSDI), December 2004.

[28] R. Sommer and V. Paxson. Enhancing byte-level network intrusion detection signatures with context. In $10^{\text {th }}$ ACM Conference on Computer and Communication Security (CCS), Washington, DC, October 2003

[29] S. Staniford, J. A. Hoagland, and J. M. McAlerney. Practical automated detection of stealthy portscans. Journal of Computer Security, 10(1/2):105-136, 2002.

[30] S. Staniford, V. Paxson, and N. Weaver. How to 0wn the internet in your spare time. In 11th USENIX Security Symposium, Aug. 2002.

[31] G. Vigna and R. Kemmerer. NetSTAT: A network-based intrusion detection system. Journal of Computer Security, 7(1):37-71, 1999.

[32] G. Vigna, W. Robertson, and D. Balzarotti. Testing networkbased intrusion detection signatures using mutant exploits. In ACM Conference on Computer and Communication Security (ACM CCS), Washington, DC, October 2004.

[33] V. Yegneswaran, P. Barford, and D. Plonka. On the design and use of internet sinks for network abuse monitoring. In Recent Advances in Intrusion Detection, Sophia Antipolis, France, Sept. 2004

[34] V. Yegneswaran, P. Barford, and J. Ullrich. Internet intrusions: Global characteristics and prevalence. In ACM SIGMETRICS, San Diego, California, June 2003.

[35] V. Yegneswaran, J. T. Giffin, P. Barford, and S. Jha. An architecture for generating semantic-aware signatures. Technical Report 1507, University of Wiscsonsin, 2004. http://www.cs.wisc.edu/ vinod/nemean-tr.pdf.

\section{Notes}

${ }^{1}$ The first labor of the Greek hero Heracles was to rid the Nemean plain of a fierce creature known as the Nemean Lion. After slaying the beast, Heracles wore its pelt as impenetrable armor in his future labors.

${ }^{2} \mathrm{~A}$ honeynet is a network of high-interaction honeypots.

${ }^{3}$ A negligible amount of non-malicious traffic on our honeynet was caused by misconfigurations and was easily separated from the malicious traffic.

${ }^{4}$ Honeyd is a popular open-source low-interaction honeypot tool that simulates virtual machines over unused IP address space.

${ }^{5}$ The check for destinations avoids hotspot misconfiguration.

${ }^{6} \mathrm{An}$ observation which was confirmed through personal communication with C. Kreibich, one of the authors of Honeycomb.

${ }^{7}$ Signature 2 is a more general version of signature 1 which is redundant.

${ }^{8}$ The Host field should be ignored. The signature would miss attacks from sources with prefixes other than 128.1. 